\title{
Analyzing QoS metrics in IEEE 802.11ah networks with traffic differentiation
}

\author{
Sergio Zumpano Arnosti ${ }^{1}$, Juliana Freitag Borin ${ }^{1}$ \\ ${ }^{1}$ Instituto de Computação - Universidade de Campinas (UNICAMP) \\ Av. Albert Einstein, 1315 - Cidade Universitária - Campinas - SP - Brazil \\ serza.arnosti@gmail.com, juliana@ic.unicamp.br
}

\begin{abstract}
The IEEE 802.11ah specification was proposed recently to provide a communication solution for Internet of Things (IoT) devices. Among the 802.11 ah related work that evaluate one of its main features, the Restricted Access Window (RAW), only a few consider Quality of Service (QoS) and the inherent traffic heterogeneity in IoT. The purpose of this paper is to compare $R A W$ scenarios including IoT devices with different traffic patterns and QoS requirements. The results suggest that the increase of medium contention inside $R A W$ slots in dense networks may be a limiting factor for providing $Q o S$.
\end{abstract}

\section{Introduction}

The embedding of small processing and communication devices in everyday objects and the integration of these with the environment lined up with the development of areas like ubiquitous computing [Weiser 1999] and machine learning has enabled the creation of the so called smart environments. Smart environments have the capacity of collecting data, processing it in the cloud, and extracting knowledge, which can be used to provide services and make people's life easier and more comfortable [Ahmed et al. 2016, Meira 2016].

Those objects will be constantly connected to the internet and, according to Cisco $^{1}, 50$ billion will be connected until 2020. This large integration of things to the internet in a transparent way is raising a new internet generation, called Internet of Things (IoT) [Ashton 2009, Ahmed et al. 2016]. A great number of challenges related to network and communication come with the IoT. Among these, it is possible to highlight the management of the large number of connected devices, energy saving, and efficient sharing of network resources. In this scenario, technologies and network protocols for wireless communications are key elements for connecting devices in IoT and also to provide people interaction with them.

The IEEE 802.11ah protocol [IEEE 2017], also known as Wi-Fi HaLow, is a solution for IoT scenarios that need high transmission rates and long signal range. Unlike the other protocols from the IEEE 802.11 set, Wi-Fi HaLow works in a sub-1GHz radio frequency. At outdoor environments, the protocol can reach up to $1 \mathrm{~km}$ of signal range with a transmission rate around $150 \mathrm{Kbps}$. At lower distances and using specific channel modulations it can reach rates from $300 \mathrm{Kbps}$ to $346 \mathrm{Mbps}$.

The protocol also introduces new mechanisms to support a large number of IoT nodes and to provide energy efficiency. One of such mechanisms, the Restricted Access

\footnotetext{
${ }^{1}$ http://www.cisco.com/web/solutions/trends/iot/portfolio.html
} 
Windows (RAW) restricts the number of devices contending for the medium simultaneously. Basically, devices are divided in RAW groups and each group has an access window divided in slots. More specifically, the 802.11ah access point (AP) sends beacons containing the parameters of the current RAW group. At each beacon interval, there is a period for the RAW group, where only the devices belonging to that group may contend for the medium and only in their assigned slots. There is also a free contention period, where all stations can contend for the medium [IEEE 2017, Wang and Fapojuwo 2017].

Figure 1 shows how the RAW mechanism works. In the example, device $d$ belongs to RAW B group and was assigned to slot $j$, where it is allowed to contend for the medium.

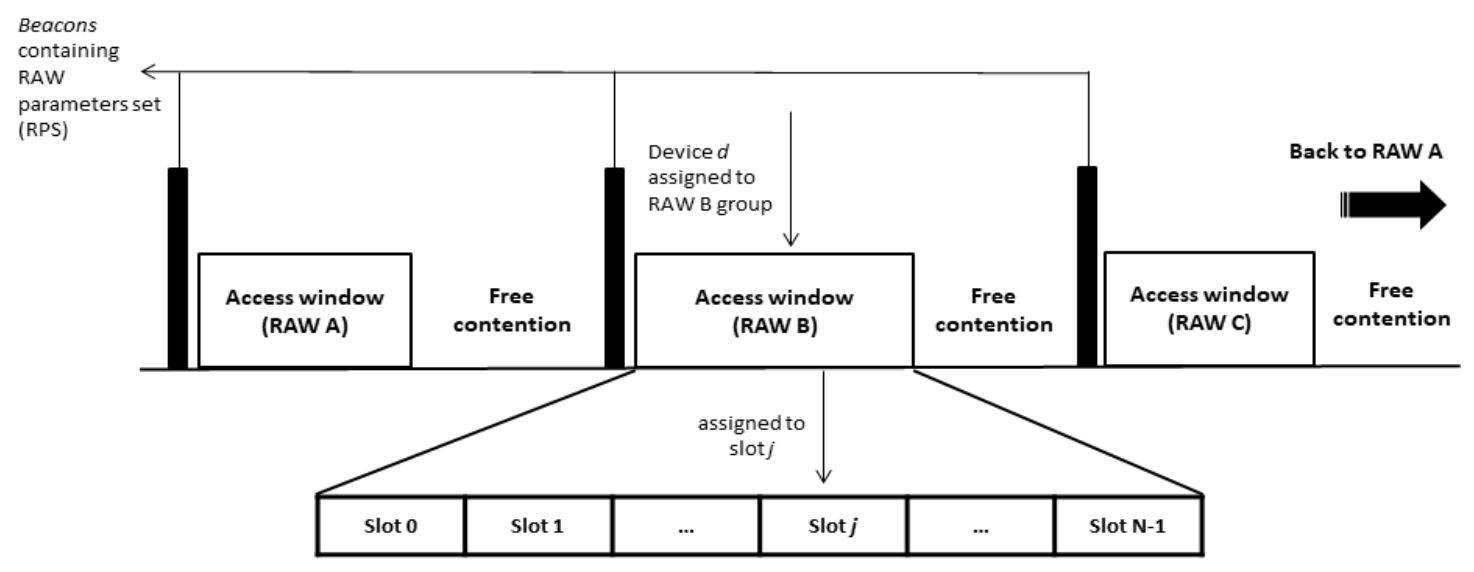

Figure 1. Example of a RAW turnaround. Adapted from [Tian et al. 2016].

The IEEE 802.11ah specification does not define a RAW grouping strategy. Therefore, in this paper, we evaluate the impact of two grouping strategies in scenarios with heterogeneous traffic: (1) mixing nodes with different traffic requirements inside the same groups and (2) creating dedicated groups for each type of traffic. In this way, the main contributions of this paper are:

- an analysis of the impact of two RAW grouping approaches on the performance of the protocol considering QoS metrics;

- insights into limiting factors for providing QoS in dense IEEE 802.11ah networks.

The reminder of the paper is organized as follows. Section 2 summarizes important related work and their results. Section 3 describes the system model. Section 4 details both RAW grouping strategies. Section 5 presents a complete description of the simulation setup, scenarios and used parameters. The simulation results are analyzed and discussed in Section 6. Finally, Section 7 concludes the paper.

\section{Related Work}

Recent studies about the IEEE 802.11ah protocol have focused in the improvement of the RAW grouping as well as in performance evaluation of the MAC layer mechanisms. In this section, we highlight some of them and their contributions.

Tian et al. [2017] proposed a real-time station grouping algorithm optimized for sensor nodes, called TAROA, which considers dynamic traffic conditions and assumes a predictable frequency in packets transmission. TAROA reveals a better performance in 
terms of throughput, resilience to dynamic traffic and better scalability when compared to the legacy Enhanced Distributed Channel Access (EDCA/DCF) mechanism.

Kim and Yeom [2017] took into account Quality of Service (QoS) and proposed an algorithm that gives priority to stations which have a high frequency of collisions. It reserves the first slot of each RAW group for those stations and adjusts the slot duration according to the total amount of network traffic. The proposed algorithm shows a delay 2.6 times lower than the standard 802.11ah.

More recently, Šljivo et al. [2018] investigated the TCP behavior evaluating the influence of RAW and TIM (Traffic Indication Map) mechanisms in two scenarios: one including only video streaming stations and another including only sensors. They consider downlink traffic, what reveal that TIM segmentation could contribute for scalability and energy saving. They also conclude that IEEE 802.11ah can support legacy internet traffic with the properly network and transport layer configurations but with a limited number of stations.

Finally, the study of Oyegbola et al. [2018] presented a Classified Medium Access Control Algorithm (CL-MAC). The algorithm implements a hybrid TDMA ${ }^{2}$ and CSMA/ $\mathrm{CA}^{3}$ scheme for channel access and allocates slots inside RAW groups based on four traffic profiles that are classified according to traffic priority and distance from AP. The result shows that CL-MAC is a better scheme than EDCA in terms of throughput for two of the considered traffic profiles.

The discussed works propose improvements to medium access control mechanisms and bring important insights about the IEEE 802.11ah performance. To the best of our knowledge, Oyegbola et al. [2018] is the only work to consider the heterogeneity of the traffic when grouping nodes running different applications, however, the solution proposed by them is not standard compliant. In contrast, the main goals of this paper are to analyze (1) the impacts on QoS metrics in network scenarios where IoT nodes with different traffic models and requirements are simultaneously contending for the medium inside the same group and (2) whether or not QoS could be improved by placing nodes with different traffic models in distinct groups using a standard-compliant strategy.

\section{System model}

The system consists of sensors and surveillance cameras scattered in the coverage area of an IEEE 802.11ah AP. Figure 2 represents a typical configuration of such a system.

Sensor nodes run a TCP client application that measures a certain magnitude and send the data every 1 second to the TCP back-end server connected to the AP. On the other hand, camera nodes run an UDP client application, which verifies for motion at intervals of 1 second and in case of detected motion, sends a 10 seconds video stream to the UDP back-end server connected to the AP. The use of these applications was inspired in the work of [Šljivo et al. 2018].

Following previous work [Baños-Gonzalez et al. 2016, Khorov et al. 2015], we considered the MCS8 channel modulation with $2 \mathrm{Mhz}$ bandwidth and with a single-

\footnotetext{
${ }^{2}$ Time Division Multiple Access

${ }^{3}$ Carrier Sense Multiple Access with Collision Avoidance
} 


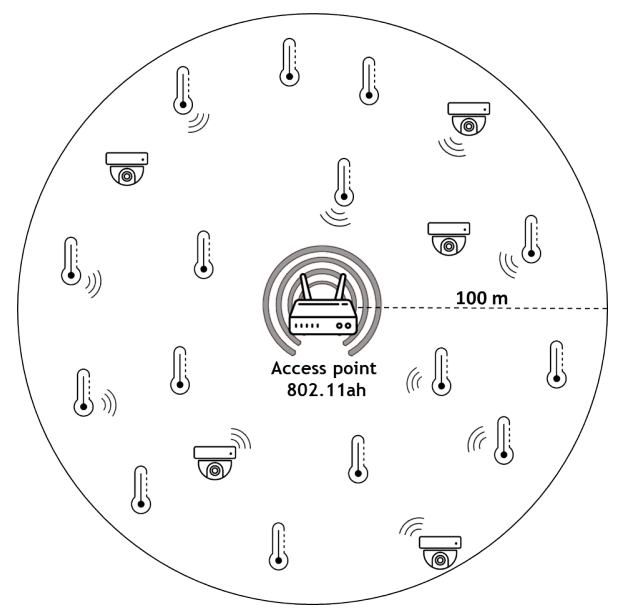

Figure 2. Configuration of the simulations.

spatial-stream. This channel modulation provides a proper data rate for both sensors and surveillance camera applications.

Regarding the RAW mechanism, we assume the zero-bit RAW slot definition format, which provides a maximum number of 63 RAW slots (RSN) per group and a Slot Duration Count (SDC) of 255. Considering that, in this paper, the RSN for a group follows the rule of Equation 1.

$$
R S N=\left\{\begin{array}{ll}
n, & \text { if } n<63 \\
63, & \text { if } n \geq 63
\end{array},\right.
$$

where $n$ is the number of nodes belonging to a specific RAW group. Also, the slot duration is calculated by Equation 2 [IEEE 2017] which is dependent on the value of SDC, described by Equation 3 .

$$
D_{R A W}^{\text {slot }}=500+(120 \times S D C), \quad \text { (2) } \quad S D C=\left\lceil\frac{T X_{\text {total }}-500}{120}\right\rceil,
$$

where $T X_{\text {total }}$ is the total duration of a packet transmission.

To calculate correctly the value of $T X_{\text {total }}$, it is important to consider the entire packet transmission at the PHY layer, as follows in Equation 4 [Šljivo et al. 2018].

$$
T X_{\text {total }}=D_{\text {pay }}+D_{p h}+S I F S+D_{a c k},
$$

where $D_{p a y}$ is the payload duration, $D_{p h}$ is the preamble and header (signal field) duration, which has a fixed duration of $240 \mu s, S I F S$ is the Short Interframe Space, equals to 160 $\mu s$, and $D_{a c k}$ is the duration of the acknowledge, equals to $680 \mu s$. The $D_{\text {pay }}$ value is calculated by Equation 5 .

$$
D_{\text {pay }}=N_{\text {symb }} \times D_{\text {symb }},
$$

where $N_{\text {symb }}$ is the number of symbols for Binary Convolutional Code (BCC) encoding and $D_{\text {symb }}$ is the symbol duration of the Orthogonal Frequency-Division Multiplexing 
(OFDM) with normal guard interval, equals to $40 \mu s$.

The $N_{\text {symb }}$ is calculated based on Equations 6 and 7 [Šljivo et al. 2018], where $D B P S$ is the data bits per second, $D R$ is the data rate in bits per second of the chosen channel modulation, $N S S$ is the number of spacial streams, equals to 1 in this paper, $D_{\text {symb }}$ is the symbol duration of OFDM and L is the packet size in bytes:

$$
D B P S=D R \times N S S \times\left(\frac{D_{\text {symb }}}{10^{-6}}\right), \quad(6) \quad N_{\text {symb }}=\left\lceil\frac{8+(L \times 8)+6}{D B P S}\right\rceil .
$$

The beacon interval, $B_{\text {int }}$, is calculated according to Equation 8 , where $D_{R A W}^{\text {group }}$ is the duration of the RAW group, calculated in Equation 9 [IEEE 2017].

$$
B_{\text {int }}=2 \times D_{R A W}^{\text {group }}, \quad \text { (8) } \quad D_{R A W}^{\text {group }}=R S N \times D_{R A W}^{\text {slot }} .
$$

\section{RAW grouping strategies}

In this paper we evaluate two RAW grouping strategies: Mixed Groups (MG), which can be taken as a baseline, where sensors and surveillance cameras are randomly distributed inside groups, and Dedicated groups (DG), where sensors and cameras are placed in different and dedicated groups.

Figures 3 and 4 illustrate the MG and DG strategies, respectively. In the MG strategy, the nodes were mixed but equally divided in a number of RAW groups and the RAW slot duration for sensors $\left(t_{s}\right)$ and cameras $\left(t_{c}\right)$ was calculated based on the largest packet transmission duration, in this case the packet generated by the camera application. The standard determines that slots inside a RAW group must have the same duration, so using a duration less then $t_{c}$ in the MG strategy would hinder cameras transmission.

In the DG strategy, the nodes were divided in dedicated groups according to their types since the protocol provides means for the nodes to inform which application they are running. RAW slots duration for groups of sensors were calculated based on the sensor packet transmission duration and for the group of cameras on the camera packet transmission duration.

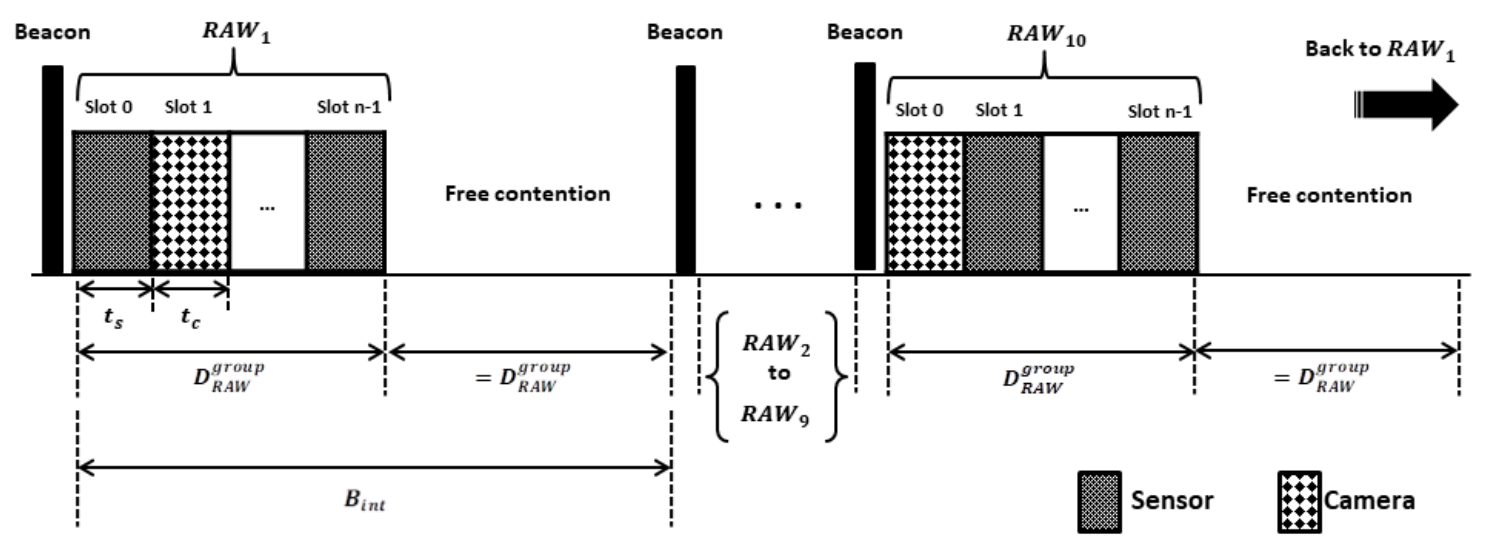

Figure 3. MG scenario, where sensors and cameras are mixed inside RAW groups. The duration of the free contention is equal to $D_{R A W}^{g r o u p}$ and for all RAW groups $t_{s}=t_{c}=$ camera packet transmission duration. 


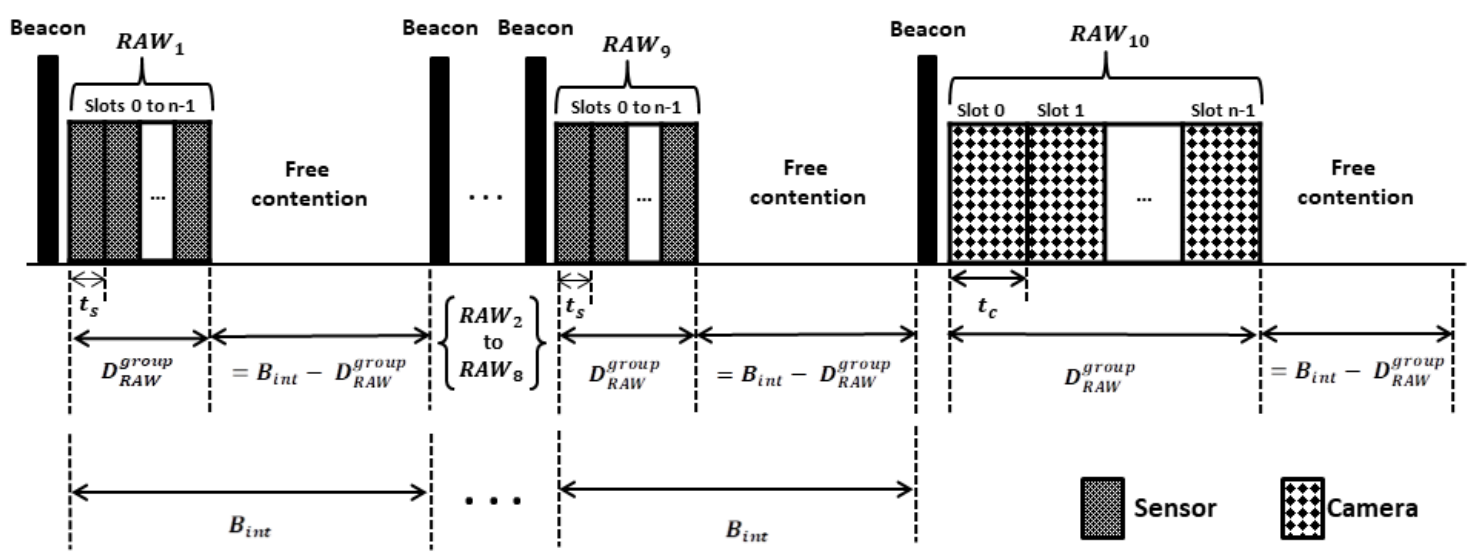

Figure 4. DG scenario, where sensors are equally distributed in the first nine RAW groups and cameras are allocated in the last $\left(R A W_{10}\right)$. The duration of the free contention is equal to the current RAW group duration, $D_{R A W}^{g r o u p}$, subtracted from beacon interval, $B_{\text {int }}$. The slot duration for sensors is $t_{s}=$ sensor packet transmission duration, while for cameras is $t_{c}=$ camera packet transmission duration.

The beacon interval for both strategies is given by $B_{\text {int }}$ (Eq. 8), however, for MG strategy, at each beacon interval, the free contention period has the same duration of the RAW group and for the DG strategy, the free contention period has the duration of $B_{\text {int }}-D_{R A W}^{\text {group }}$.

\section{Simulation setup and methodology}

The performance evaluation was based on simulation. The simulations were performed using NS- $3^{4}$ version 3.23 and the 802.11 ah module implemented in [Tian et al. 2016] and extended in [Tian et al. 2018]. The nodes were randomly distributed within a radius of 100 meters around the access point (AP), which runs the application servers. That radius allows all nodes to keep a good enough channel condition to associate with the AP and to take advantage of the $7.8 \mathrm{Mbps}$ provided by the MCS8 modulation.

We ran ten independent replications for each different number of nodes $(80,160$, 320,640 , and 1280) and for both grouping strategies. The nodes were randomly generated with a $90 \%$ probability of being a sensor and $10 \%$ of being a camera. To the best of our knowledge, there are no references showing an adequate proportion for the chosen devices; we believe that the chosen values are representative of a future smart city scenario. The nodes were divided in ten RAW groups with one group per beacon interval.

In order to analyze the impact of the two grouping strategies on the performance of the protocol and to compare them in terms of QoS, we considered the following metrics: end-to-end delay, jitter, data/packet loss, number of cancelled transmissions due to crossing the RAW slot boundary and throughput.

To enable the replication of the experiments, the remainder of this section presents all the parameters used to configure the simulations.

\footnotetext{
${ }^{4}$ https://www.nsnam.org/
} 


\subsection{Summary of the simulation parameters}

The parameters passed for simulator and for the 802.11ah module in these experiments are presented in Table 1.

Table 1. Simulator and 802.11ah module parameters

\begin{tabular}{lc}
\hline Parameter & Value \\
\hline Simulation Time & $90 \mathrm{~s}$ \\
Seed & 1 \\
Run Seed & {$[1,10]$} \\
Data Mode & MCS2_8 \\
Data Rate & $7.8 \mathrm{Mbps}$ \\
Bandwidth & $2 \mathrm{Mhz}$ \\
Radius & $100 \mathrm{~m}$ \\
Page Period & 1 \\
Page Slice Length & 31 \\
Page Slice Count & 0 \\
\hline \hline
\end{tabular}

To guarantee ten independent replications for each number of nodes, we follow the recommendations about seeding and independent replications at the NS-3 manual ${ }^{5}$, so we fixed the Seed parameter and varied the Run Seed from 1 to 10. Page parameters refer to TIM segmentation mechanism and the used values configure the basic form of it, once this is not the focus of this paper. Therefore, the values define a single TIM page per beacon interval. Details of TIM segmentation can be found in [Šljivo et al. 2018] and in the standard itself [IEEE 2017].

The physical and MAC parameters values used for nodes in all simulations are presented in Table 2. For the AP, some values for PHY layer are different: Tx Power Start/End must be adjusted to $30.0 \mathrm{dBm}$; Tx and Rx Gain must be adjusted to $3.0 \mathrm{~dB}$. Some of the propagation loss model parameters are based on [Hazmi et al. 2012] and in the low-power 802.11ah radio hardware prototype developed by [Ba et al. 2016].

For the MAC layer it is important to activate the QoS Support to enable the use of Enhanced Distributed Channel Access (EDCA), consequently the use of the access categories and priority queues, in this case, the AC_BE (Best Effort) and the AC_VI (Video). These traffic categories were chosen to promote traffic differentiation.

The IEEE 802.11ah simulation module was adapted to insert an access category tag in the packets for directing them to the AC_BE queue in the case of sensors and to the AC_VI queue in the case of cameras. The packet size for the sensor application is 100 bytes and for the video applications is 1500 bytes [Baños-Gonzalez et al. 2016]. Table 3 shows the main application parameters.

The RAW group configuration follows a RAW parameter set (RPS) provided in an input file for the simulator. The file may include multiple RPS and its parameters are described in Table 4. The format of the RAW configuration file is based on the extended implementation of [Tian et al. 2018].

\footnotetext{
${ }^{5} \mathrm{https} / / /$ www.nsnam.org/docs/manual/html/random-variables.html
} 
Table 2. PHY and MAC layer parameters

\begin{tabular}{|c|c|c|c|}
\hline PHY Parameter & Value & MAC Parameter & Value \\
\hline Frequency & $900 \mathrm{Mhz}$ & Access Categories & $\mathrm{AC} \_\mathrm{BE}, \mathrm{AC}_{-} \mathrm{VI}$ \\
\hline Tx Power Stard/End & $0 \mathrm{dBm}$ & QoS Support & Enabled \\
\hline Tx and Rx Gain & $0 \mathrm{~dB}$ & Active Probing & Disabled \\
\hline Tx Power Levels & $1 \mathrm{~dB}$ & Rate Ctrl. Algorithm & ConstantRate \\
\hline $\begin{array}{l}\text { Noise Figure } \\
\text { Coding Method }\end{array}$ & $\begin{array}{r}6.8 \mathrm{~dB} \\
\mathrm{BCC}\end{array}$ & \multirow{2}{*}{$\begin{array}{l}\text { AC BE Queue } \\
\text { Max delay } \\
\text { Max packet number }\end{array}$} & \multirow[b]{2}{*}{$\begin{array}{r}400 \mathrm{~ms} \\
400\end{array}$} \\
\hline Propag. Loss Model & LogDistance & & \\
\hline $\begin{array}{l}\text { Exponent } \\
\text { Ref. Loss }\end{array}$ & $\begin{array}{r}3.76 \\
8.0 \mathrm{~dB}\end{array}$ & \multirow{3}{*}{$\begin{array}{l}\text { AC_VI Queue } \\
\text { Max delay } \\
\text { Max packet number }\end{array}$} & \multirow{3}{*}{$\begin{array}{r}200 \mathrm{~ms} \\
400\end{array}$} \\
\hline Ref. Distance & $\begin{array}{r}8.0 \mathrm{~dB} \\
1.0\end{array}$ & & \\
\hline Propag. Delay Model & ConstantSpeed & & \\
\hline Error Rate Model & YansErrorRate & & \\
\hline
\end{tabular}

Table 3. Sensor and Camera Applications parameters and characteristics

\begin{tabular}{|c|c|c|c|}
\hline Sersor App & Value & & \\
\hline Access Category & AC_BE & Camera App & Value \\
\hline Packet Size & 100 bytes & Access Category & $\overline{A C_{-} V I}$ \\
\hline Traffic Interval & $1.0 \mathrm{~s}$ & Packet Size & 1500 bytes \\
\hline Transport Protocol & $\overline{\mathrm{TCP}}$ & Motion Verification Interval & $1.0 \mathrm{~s}$ \\
\hline Min RTO & $500 \mathrm{~ms}$ & Motion Duration & $10.0 \mathrm{~s}$ \\
\hline Segment Size & 616 & Motion Probability & $70 \%$ \\
\hline Delay Ack Count & $\begin{array}{r}010 \\
0\end{array}$ & $\underline{\text { Data Rate }}$ & $128 \mathrm{~kb} / \mathrm{s}$ \\
\hline Data Retries & 18 & Transport Protocol & UDP \\
\hline ReTx Threshold & 2 & & \\
\hline
\end{tabular}

Table 4. RAW Parameter Set (RPS) for each Raw Group

\begin{tabular}{|c|c|c|}
\hline RAW Parameter & Value & Meaning \\
\hline RAW Control & 0 & $\begin{array}{l}\text { RAW group can be accessed by any nodes within the } \\
\text { RAW group. }\end{array}$ \\
\hline Cross Slot Boundary & 0 & Node cannot cross the slot boundary. \\
\hline Slot Format & 0 & $\begin{array}{l}\text { Define } 6 \text { bits for Raw Slot Number (max.: } 63 \text { slots) } \\
\text { and } 8 \text { bits for Slot Duration Count (max.: 255). }\end{array}$ \\
\hline Slot Duration Count & $S D C$ & Used to calculate the slot duration. \\
\hline Raw Slot Number & $R S N$ & Number of slots in the RAW group. \\
\hline Page & 0 & Page index of the subset of AIDs. \\
\hline AID Start & $A I D_{S}$ & Node with lowest AID allocated in the RAW group. \\
\hline AID End & $A I D_{E}$ & Node with highest AID allocated in the RAW group. \\
\hline
\end{tabular}




\section{Results analysis}

In this section we analyze the data collected from the simulation experiments. For each metric, we present the results related to sensor and camera nodes. Plotting the collected data for delay, jitter, number of cancelled transmissions, and throughput, we observed that the distribution was skewed. Therefore, since the data doesn't follow a normal distribution, we use the median as a statistical parameter to represent the set of data. The lines were plotted to represent a trend for scenarios with different number of nodes.

(a)

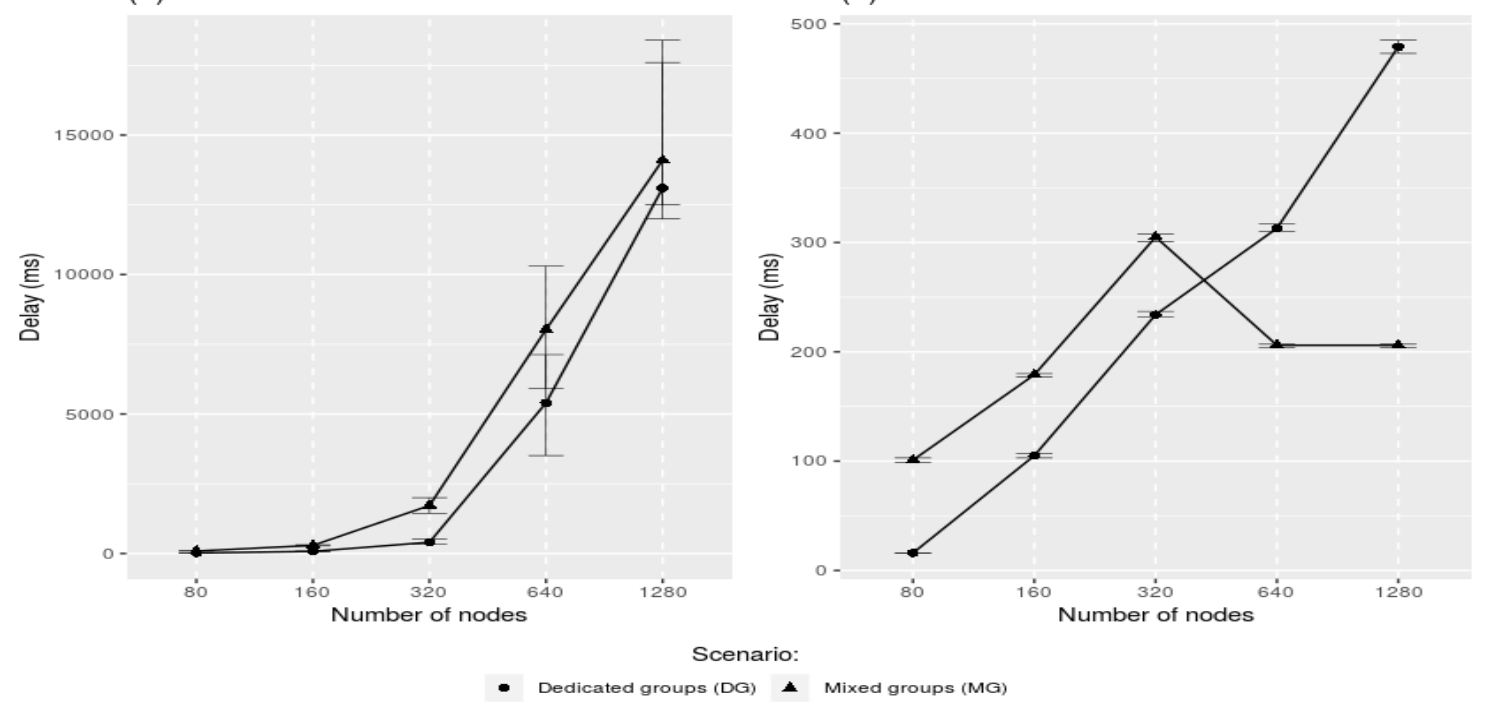

Figure 5. Median packet delay for sensors (a) and cameras (b).

We begin discussing the results for end-to-end packet delay metric. Figure 5(a) shows the median packet delay for sensor nodes. The increasing behavior of the delay as the number of nodes increases was also observed in a sensor only network in [Tian et al. 2017]. Still in Figure 5(a), for simulations with 80, 160 and 320 nodes, DG scenario presents lower median delay than MG. With 320 nodes we can note the higher difference between scenarios with a median of $1720 \mathrm{~ms}$ for $\mathrm{MG}$ and $403 \mathrm{~ms}$ for DG, a decrease of $76.6 \%$. For 640 and 1280 nodes, there is no statistical difference.

Figure 5(b) depicts the median packet delay for camera nodes. The DG scenario shows a significant reduction of $84.2 \%, 41.3 \%$ and $23.3 \%$ in the median packet delay for 80, 160 and 320 nodes, respectively. With 640 and 1280 nodes, MG scenario shows a big decrease in median delay for cameras reaching $57 \%$ for 1280 nodes.

Once there are more nodes than available slots in the groups when the total number of nodes is 640 and 1280, two or more nodes can be assigned to the same RAW slot what causes the nodes to contend for the medium inside the slots. Therefore, since in the MG scenario the RAW slot duration is the same for all groups and as large as the bigger packet transmission duration (camera packets), more than one sensor node can get the access to the medium and transmit a packet in the same slot, consequently reducing the number of nodes contending in the free contention period what could reduce the end-to-end delay for camera packets. Contrarily, this does not happen in the DG scenario, because the slots fit the size relative to the packet transmission duration of that group type and if the node 
could not get access in its slot, it will have another opportunity to transmit only in the free contention periods or on the next occurrence of its RAW slot.

The high packet loss rate for camera nodes in the MG scenario (Figure 7) may also explain the delay results when the number of nodes is 640 and 1280. Packets dropped due to deadline violation are not considered in the delay calculation what may be leading to a better delay result when compared to the DG scenario. These scenarios will be further explored and investigated in the future work.

(a)

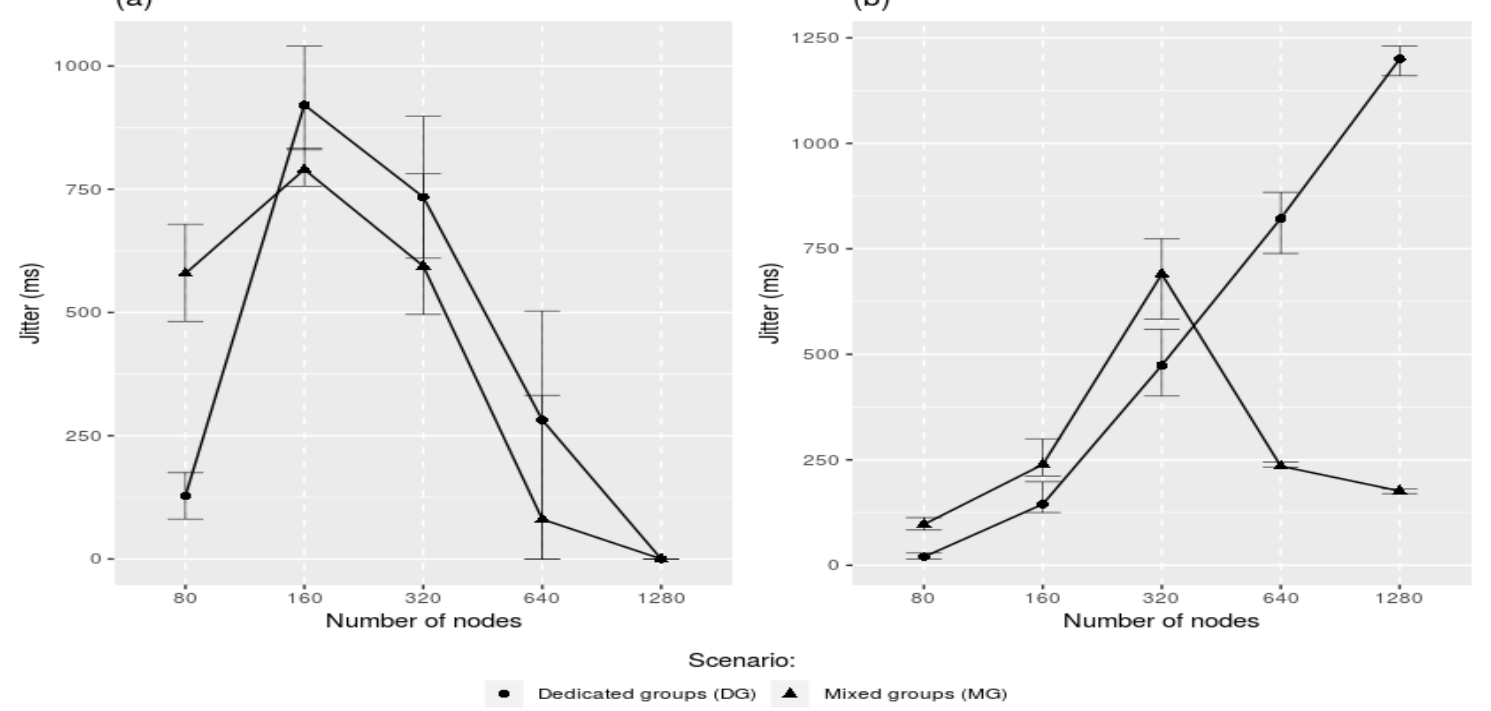

Figure 6. Median jitter for sensors (a) and cameras (b).

Figure 6 shows the median jitter (delay variation), which was calculated by NS3 according to [Schulzrinne et al. 2003]. The jitter for sensors is not so relevant if the application is delay tolerant. Nevertheless, for real time applications this could be crucial. In Figure 6(a) there is statistical difference only for 80 nodes.

In contrast, for cameras, jitter is an important metric, because a high jitter could lead to an irregular reception of the packets and consequently a bad experience for the receptor and information loss. In Figure 6(b) it is possible to note a substantial decrease of the jitter for DG with 80, 160 and 320 nodes, which in the best case reduced from $690 \mathrm{~ms}$ to $474 \mathrm{~ms}, 31.3 \%$. Differently, when nodes start to contend in the same slot, MG scenario with larger slots duration is the best approach. DG scenario shows a high median jitter of $1200 \mathrm{~ms}$ against $176 \mathrm{~ms}$ of MG for 1280 nodes.

Another important metric for analyzing QoS is the data/packet loss (Figure 7). As sensor nodes use TCP, we measured loss in bytes, called as 'data loss' and as cameras use UDP, we measured loss in packets, called 'packet loss'. For sensors, it is possible to see in Figure 7(a) that for 80 and 160 nodes the MG scenario loses more bytes than DG. When the number of nodes increase, there is an inversion, the MG scenario shows better results losing less data, but at the same time, as the total number of nodes increase the total data loss increase. Analyzing the results for cameras in Figure 7(b), the DG scenario presents better results for all number of nodes, in other words, DG lost less packets than MG. In the best case, for 80 nodes, there is a difference of 22.9 percentage points and in 
(a)

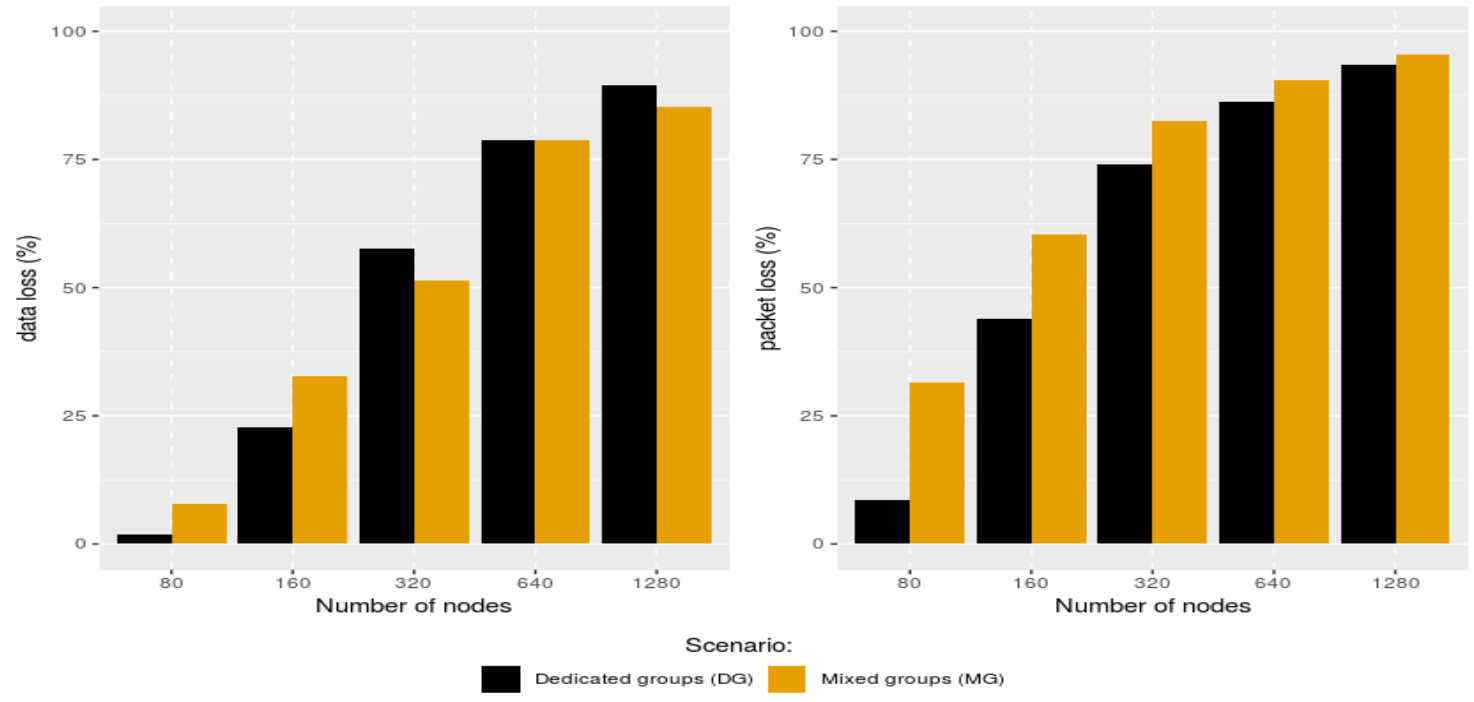

Figure 7. Mean data loss for sensors (a) and mean packet loss for cameras (b).

the worst case, for 1280 nodes, of 2.0 percentage points. It is evident from Figure 7 that system saturates with 640 and 1280 nodes.

Specially for cameras, this huge packet loss when we increase the number of nodes in the network could be a serious problem for the protocol, because for 1280 nodes with Dedicated groups (DG), 93.3\% represents a high deficit of important information reception in a surveillance system. Despite the sensor nodes take advantage of the TCP retransmission mechanism, until the end of the 90 seconds of the simulation, for 1280 nodes in the MG scenario, the AP could only receive $14.8 \%$ of the total transmitted information.

(a)

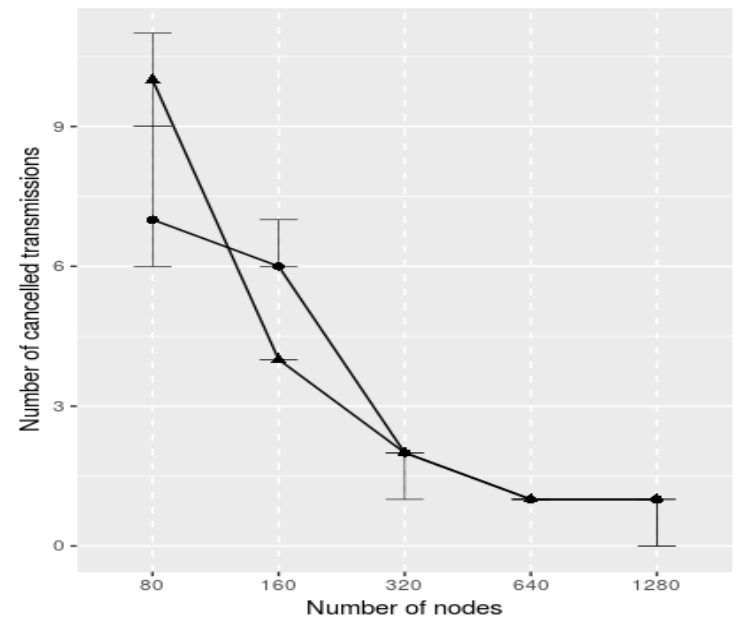

(b)

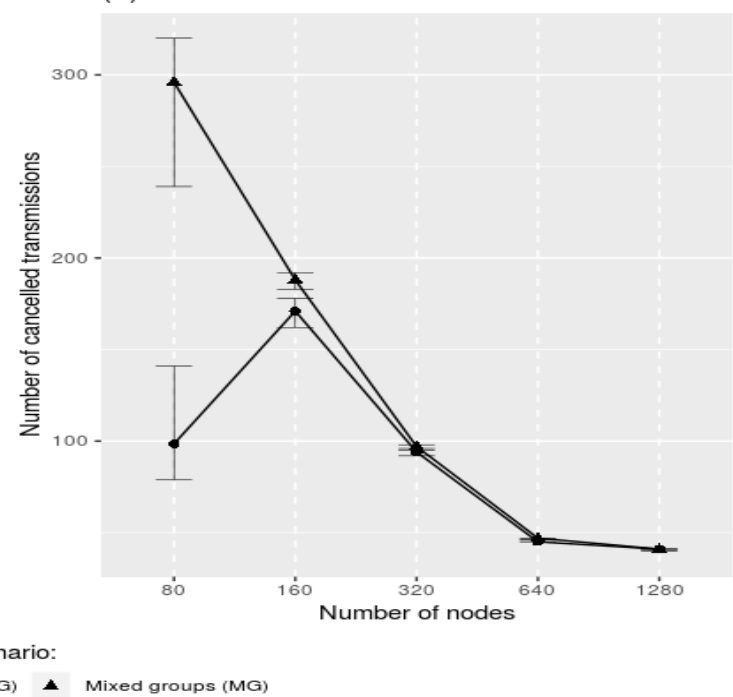

Figure 8. Median cancelled transmissions due to crossing slot boundary for sensors (a) and for cameras (b).

In this work, nodes transmission cannot cross the slot boundary (RAW option) and when this will happen the transmission is cancelled. To investigate whether or not this 
option affects the data/packets loss, we analyzed the number of cancelled transmissions (Figure 8). Differently than expected, the number of cancelled transmissions decrease as the number of nodes in the network increase. For sensors (Figure 8(a)), there is no statistical different among the two scenarios, whereas for cameras (Figure 8(b)) the number of cancelled transmissions decreases a lot in DG when simulated with 80 nodes, but with 160 this number increases, being slightly better than MG and decreases for the following numbers of nodes. Considering that, we can infer that the causes of the increasing in data/packets loss with the escalation of nodes is exclusively related to the high medium contention. With 640 and 1280 nodes, they start to contend for the medium not only in the free period, but also inside the slots.

(a)

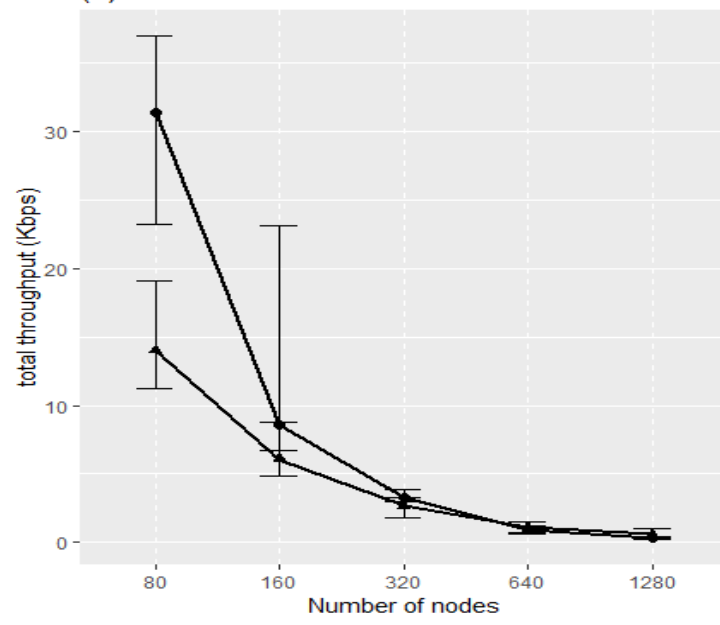

(b)

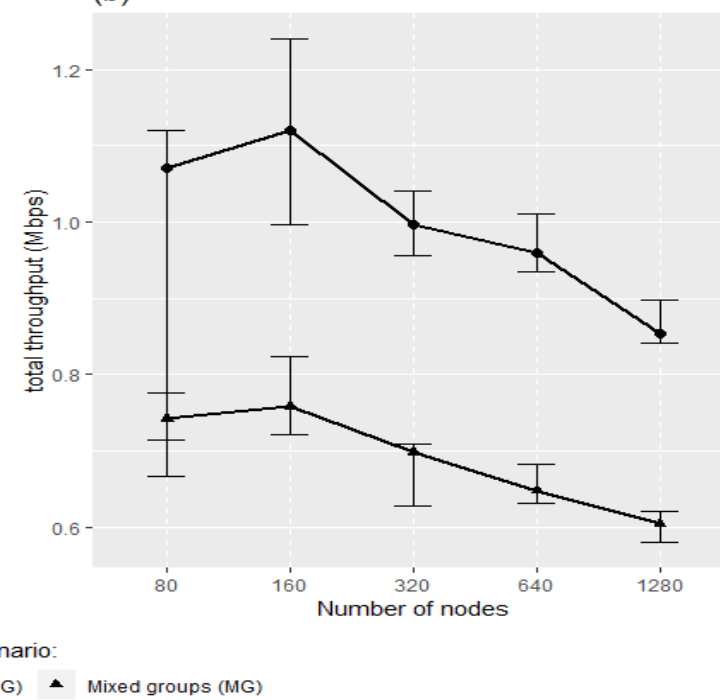

Figure 9. Median of the total throughput for sensors (a) and for cameras (b).

That high loss of packets could impact on the total throughput of the applications, Figure 9 shows the total throughput for the two applications, sensors and cameras. For sensors, Figure 9(a), the DG scenario presents an increase of $224.3 \%$ in the total throughput for 80 nodes, but for the others numbers of nodes there is no statistical difference. The graph also shows a decrease tendency as the number of nodes increase, falling drastically from $31.4 \mathrm{Kbps}$ with 80 nodes to $8.6 \mathrm{Kbps}$ when we double the number of nodes in the DG. With 1280 nodes, the sensors have a very low total throughput, reaching a rate below $1 \mathrm{Kbps}$ for both scenarios, DG and MG.

On the other hand, for cameras in Figure 9(b) we can note that for 160, 320, 640 and 1280 nodes the DG scenario shows better values for total throughput if compared to MG. In DG, the high median of the total throughput is $1.12 \mathrm{Mbps}$ for 160 nodes, while the low value is $0.85 \mathrm{Mbps}$ for 1280 , a reduction of $24.1 \%$.

\section{Conclusion}

This paper analyzed the impact of two IEEE 802.11ah standard compliant RAW grouping strategies on the QoS of heterogeneous IoT applications. In the MG strategy sensors and surveillance cameras were mixed in the same groups whereas in the DG strategy different groups were created for both applications. 
Results for cameras show that the MG scenario with larger slots significantly reduced delay and jitter in dense networks what may be a reflection of the high packet loss. For sensors, the DG scenario shows a slight decrease in end-to-end packet delay. In terms of packet loss, for cameras, DG scenario shows an improvement, but for both scenarios, DG and MG, the loss is very high, staying above $75 \%$ for dense networks. Last but not least, throughput for cameras is significantly increased in DG scenario.

We can conclude that among the analyzed strategies, there is not a better solution for both applications. However, considering the metrics individually, DG is the best strategy for cameras in terms of delay and jitter when considering less dense networks (up to 320 nodes), and presents better results in terms of packet loss and a great improvement in throughput. For sensors, in terms of delay, jitter and throughput, the strategies could be considered equivalent, but MG turned out better in terms of data loss for most of the considered number of nodes.

Finally, since IoT will have a huge number of devices per access point, our results also suggest that the increase in network density and contention inside RAW slots may limit QoS provisioning in terms of delay, jitter and packet reception. Accordingly, modifications in the legacy mechanisms for channel access together with the development of grouping algorithms for networks with heterogeneous devices is of paramount importance for the improvement of the 802.11ah protocol.

\section{Acknowledgement}

The authors would like to thank the financial support provided by the Coordenação de Aperfeiçoamento de Pessoal de Nível Superior - Brasil (CAPES) - Finance Code 001; and Amina Šljivo from the Department of Information Technology of Ghent University for the technical support with the 802.11ah module.

\section{References}

Ahmed, E., Yaqoob, I., Gani, A., Imran, M., and Guizani, M. (2016). Internet-of-thingsbased smart environments: state of the art, taxonomy, and open research challenges. IEEE Wireless Communications, 23(5):10-16.

Ashton, K. (2009). That 'Internet of Things' Thing. RFID Journal.

Ba, A., Liu, Y., van den Heuvel, J., Mateman, P., Busze, B., Gloudemans, J., Vis, P., Dijkhuis, J., Bachmann, C., Dolmans, G., Philips, K., and de Groot, H. (2016). 26.3 a 1.3nj/b IEEE 802.11ah fully digital polar transmitter for IoE applications. In 2016 IEEE International Solid-State Circuits Conference (ISSCC), pages 440-441.

Baños-Gonzalez, V., Afaqui, M. S., Lopez-Aguilera, E., and Garcia-Villegas, E. (2016). IEEE 802.11ah: A technology to face the IoT challenge. Sensors, 16(11).

Hazmi, A., Rinne, J., and Valkama, M. (2012). Feasibility study of IEEE 802.11ah radio technology for IoT and M2M use cases. In 2012 IEEE Globecom Workshops, pages 1687-1692.

IEEE (2017). IEEE standard for information technology-telecommunications and information exchange between systems - local and metropolitan area networks-specific requirements - part 11: Wireless lan medium access control (MAC) and physical 
layer (PHY) specifications amendment 2: Sub $1 \mathrm{ghz}$ license exempt operation. IEEE Std 802.11ah-2016 (Amendment to IEEE Std 802.11-2016, as amended by IEEE Std 802.11ai-2016), pages 1-594.

Khorov, E., Lyakhov, A., Krotov, A., and Guschin, A. (2015). A survey on IEEE 802.11ah: An enabling networking technology for smart cities. Computer Communications, 58:53 - 69. Special Issue on Networking and Communications for Smart Cities.

Kim, J. and Yeom, I. (2017). QoS enhanced channel access in IEEE 802.11ah networks. In 2017 17th International Symposium on Communications and Information Technologies (ISCIT), pages 1-6.

Meira, S. (2016). "SINAIS do FUTURO IMEDIATO, \#1: internet das coisas", Ikewai. Online: http://www.ikewai.com/WordPress/2016/12/12/sinais- do-futuro-imediato-1internet-das-coisas/. Acessed: 2017-Feb-17.

Oyegbola, K., Zubair, S., Onwuka, E., and Ramat, Y. (2018). Classified medium access control algorithm (CL-MAC)for enhanced operation of IEEE 802.11ah. Covenant Journal of Informatics \& Communication Technology, 6(1):44 - 56.

Schulzrinne, H., Casner, S. L., Frederick, R., and Jacobson, V. (2003). RTP: A Transport Protocol for Real-Time Applications. RFC 3550.

Tian, L., Deronne, S., Latré, S., and Famaey, J. (2016). Implementation and validation of an IEEE 802.11ah module for ns-3. In Proceedings of the Workshop on Ns-3, WNS3 '16, pages 49-56, New York, NY, USA. ACM.

Tian, L., Khorov, E., Latré, S., and Famaey, J. (2017). Real-time station grouping under dynamic traffic for IEEE 802.11ah. Sensors, 17(7).

Tian, L., Šljivo, A., Santi, S., De Poorter, E., Hoebeke, J., and Famaey, J. (2018). Extension of the IEEE 802.11ah ns-3 simulation module. In Proceedings of the 10th Workshop on Ns-3, WNS3 '18, pages 53-60, New York, NY, USA. ACM.

Wang, H. and Fapojuwo, A. O. (2017). A survey of enabling technologies of low power and long range machine-to-machine communications. IEEE Communications Surveys Tutorials, 19(4):2621-2639.

Weiser, M. (1999). The computer for the 21st century. SIGMOBILE Mob. Comput. Commun. Rev., 3(3):3-11.

Šljivo, A., Kerkhove, D., Tian, L., Famaey, J., Munteanu, A., Moerman, I., Hoebeke, J., and De Poorter, E. (2018). Performance evaluation of IEEE 802.11ah networks with high-throughput bidirectional traffic. Sensors, 18(2). 